\title{
Multi-epoch analysis of the $X$-ray spectrum of the active galactic nucleus in NGC 5506
}

\author{
Shangyu Sun ${ }^{1}$, Matteo Guainazzi ${ }^{2}$, Qingling $\mathrm{Ni}^{1}$, Jingchun Wang ${ }^{1}$, Chenyang Qian ${ }^{1}$,
}

\author{
Fangzheng Shi ${ }^{1}$, Yu Wang ${ }^{1}$, Cosimo Bambi ${ }^{1,3 \star}$ \\ ${ }^{1}$ Center for Field Theory and Particle Physics and Department of Physics, Fudan University, 220 Handan Road, Shanghai 200433, China \\ ${ }^{2}$ ESTEC/ESA, Keplerlaan 1, 2201AZ Noordwijk, The Netherlands \\ ${ }^{3}$ Theoretical Astrophysics, Eberhard-Karls Universität Tübingen, Auf der Morgenstelle 10, 72076 Tübingen, Germany
}

13 April 2017

\begin{abstract}
We present a multi-epoch X-ray spectroscopy analysis of the nearby narrow-line Seyfert I galaxy NGC 5506. For the first time, spectra taken by Chandra, XMM-Newton, Suzaku, and NUSTAR - covering the 2000-2014 time span - are analyzed simultaneously, using state-ofthe-art models to describe reprocessing of the primary continuum by optical thick matter in the AGN environment. The main goal of our study is determining the spin of the supermassive black hole (SMBH). The nuclear X-ray spectrum is photoelectrically absorbed by matter with column density $\simeq 3 \times 10^{22} \mathrm{~cm}^{-2}$. A soft excess is present at energies lower than the photoelectric cut-off. Both photo-ionized and collisionally ionized components are required to fit it. This component is constant over the time-scales probed by our data. The spectrum at energies higher than $2 \mathrm{keV}$ is variable. We propose that its evolution could be driven by fluxdependent changes in the geometry of the innermost regions of the accretion disk. If we take this hypothesis into account, the black hole spin in NGC 5506 is constrained to be comprised between $0.91<a<0.98$ at $90 \%$ confidence level for one interesting parameter.
\end{abstract}

Key words: Galaxies: active - Galaxies: Individual: NGC 5506 - Accretion, accretion discs

\section{INTRODUCTION}

NGC 5506 is a nearby $(z=0.0062 ; \sim 24 \mathrm{Mpc})$ edge-on Sa galaxy within the local Virgo supercluster, hosting an active galactic nucleus (AGN) classified as radio-quiet narrow-line Seyfert I (NLSy1; Nagar et al. 2002). The NLSy1 galaxies generally have lower SMBH masses and higher accretion rates (Komossa \& Xu 2007). NGC 5506 is an obscured AGN but still Compton-thin $\left(N_{H} \approx\right.$ $3 \times 10^{22} \mathrm{~cm}^{-2}$; Wang et al. 1999). Its brightness in X-ray makes it a proper source for studying in detail the high-energy activities near an active SMBH of this kind.

The hard X-ray $(E>2 \mathrm{keV})$ emission from an radio-quiet AGN (without any prominent jet) originates primarily from the vicinity of the central engine, the $\mathrm{SMBH}$, as close as a few tens of gravitational radii, $r_{\mathrm{g}}$ away from the event horizon $\left(r_{\mathrm{g}}=G M c^{2}\right.$; gravitational radius) as suggested by the short variability timescales in hard X-ray band (e.g., Grandi et al. 1992; McHardy et al. 2006; Uttley 2007; Zoghbi et al. 2013). In this innermost region of AGN, the accretion disc (AD), whose blackbody radiation spec-

^ Corresponding author: bambi@fudan.edu.cn trum peaks around ultra-violet (UV) wavelengths, is illuminated by a hard X-ray source. The spectrum of this hard X-ray source is typically described by a power-law function with a highenergy cutoff. The hard X-ray source can be a magnetically dominated corona residing above the surface of the disc (e.g., Galeev et al. 1979; Haardt \& Maraschi 1991; Haardt et al. 1994; Di Matteo 1998; Merloni \& Fabian 2001). Compton scattering, fluorescence and photoelectric absorption occur in the $\mathrm{AD}$, creating the so called "reflection spectrum"(George \& Fabian 1991). The $\mathrm{AD}$ thermal photons are also inverse-Compton up-scattered by relativistic electrons. Iron fluorescence in a dense and relatively cold medium (e.g., Barr et al. 1985; Nandra et al. 1989; Pounds et al. 1990; George \& Fabian 1991; Nandra \& Pounds 1994) can bring an emission line at about $6.4 \mathrm{keV}$. The shape of the $\mathrm{Fe} \mathrm{K} \alpha$ line includes the information of the Doppler shifts caused by the reflection if produced in a relativistically rotating $\mathrm{AD}$, the photon propagation in a curved space-time and gravitational redshift caused by the (possibly fast spinning) SMBH (Fabian et al. 1989; Laor 1991). These relativistically broadened Fe K $\alpha$ lines in AGN spectra have been resolved by Chandra, XMM-Newton, Suzaku, and NuSTAR (e.g., Ballantyne et al. 2003; Reynolds \& Nowak 2003; 
Miller 2007; Nandra et al. 2007; Fabian et al. 2009; Nardini et al. 2012; Walton et al. 2012, 2013; Risaliti et al. 2013; Parker et al. 2014; Madsen et al. 2015). The spectral analysis of relativistically broadened lines can provide a measurement of the black hole spin (e.g., Brenneman \& Reynolds 2006; Reynolds \& Fabian 2008; Parker et al. 2014) and potentially even test Einstein's gravity (Bambi 2015; Jiang et al. 2015).

In the soft X-ray band, an excess over the extrapolation of the absorbed nuclear emission has been observed in many obscured AGN spectra. The origin of this soft excess is complex. The AGN nuclear emission can heat up its circumnuclear gas, and can result in narrow radiative recombination continua (RRC) as well as recombination lines of highly-ionised atomic species. This kind of photoionized spectra have been found in several sources, e.g., the Circinus Galaxy (Sambruna et al. 2001), Mrk 3 (Sako et al. 2000; Bianchi et al. 2005; Pounds \& Page 2005), NGC 1068 (Kinkhabwala et al. 2002; Young et al. 2001), NGC 4151 (Schurch et al. 2004). In a XMM-Newton survey of obscured AGNs (Guainazzi \& Bianchi 2007), photoionization signature was found in $36 \%$ of the sample. The line widths of RRC show plasma temperatures of $\sim \mathrm{eV}$ (Kinkhabwala et al. 2002). In the spectra of some obscured AGNs, higher order transitions are enhanced with respect to pure photoionization. In these cases, resonant scattering is important in the balance between photoionization and photoexcitation. Alternatively, shocks produced by AGN outflows (King 2005) or starburst (Storchi-Bergmann et al. 1998; González Delgado et al. 2001) can also heat up ambient gas to $\sim 10^{6} \mathrm{~K}$. A spectrum of collisionally ionized plasma is characteristic for these scenarios (Viegas-Aldrovandi \& Contini 1989), but it contributes to observed soft X-ray emission only in galaxies with strong nuclear star formation (Guainazzi et al. 2009).

Bianchi et al. (2003) analyzed NGC 5506 X-ray spectra taken from 1997 to 2002, reporting only a narrow Fe K $\alpha$ line. However, deeper XMM-Newton observations unveiled a moderately broadened component of the $\mathrm{Fe} \mathrm{K} \alpha$ line (Guainazzi et al. 2010a). Furthermore, the spectrum observed by NUSTAR, which has a high sensitivity above $10 \mathrm{keV}$, constrained the high-energy cutoff of the continuum (Matt et al. 2015). In this paper, we analyze for the first time all the good quality X-ray archival data of NGC 5506. The main goal of this paper is achieving the most accurate determination so far of the relativistic accretion disk and black hole parameters, taking advantage of the unprecedented combination of signalto-noise ratio and energy broadband coverage $(0.2-79 \mathrm{keV})$.

In Sect. 2, we report the observations and data products from each instrument. In Sect. 3, we show the procedures and results on the spectral analysis. Then we discuss and conclude in Sect. 4.

\section{OBSERVATIONS AND DATA PRODUCTS}

For the sake of this work, 11 deep observations (exposure time $>20 \mathrm{ks}$ ) in X-rays were selected from NASA's High Energy Astrophysics Science Archive Research Center (HEASAC). The observation time, id, exposure time, start time, and its corresponding epoch name used in this work (E1-E11) are listed in Table 1.

\section{$2.1 \quad X M M-N e w t o n$}

The data from European Photon Imaging Camera (EPIC)Metal Oxide Semi-conductor (MOS; Turner et al. 2001) and pn (Strüder et al. 2001) were reduced using the software SAS provided by ESA (Gabriel et al. 2004). Flaring particle background was filtered using light curves extracted at high energies (Principal Investigator (PI) $>10 \mathrm{keV}$ for MOS and PI between $10 \mathrm{keV}$ and $12 \mathrm{keV}$ for $\mathrm{PN}$ ) and with single events only. We removed the high particle background intervals above the appropriate count rate thresholds (see Table 2) individually set for each observation and each instrument (Guainazzi et al. 2010a). We selected single, double, triple and quadruple events for MOS; single and double events for PN. Source spectra were extracted from circular regions around the source centroid. For MOS data, background spectra were extracted from annulus region with no extra sources around the same centroid as the corresponding source spectra. For PN, background spectra were extracted from the circular regions at the same height in detector coordinates to ensure the same charge transfer inefficiency correction as the source spectra. The redistribution matrix and the effective area were computed through the SAS tasks, ARFGEN and RMFGEN. The spectral energy range used in this work is $1-10 \mathrm{keV}$.

Data from reflection grating spectrometer (RGS) were processed with SAS v.14.0.0, by using the standard task pipeline RGSPROC. The spectral energy range used in this work is $0.2-2 \mathrm{keV}$.

\subsection{Suzaku}

The Suzaku data were reduced by software package HEASOFT v6.16, using the AEPIPELINE script following the Suzaku ABC Guide $^{1}$ to perform reprocessing (update energy calibration) and rescreening.

As for the X-ray imaging spectrometer (XIS) data, we extract spectra using a $260^{\prime \prime}$ region around the source through XSELECT. The ancillary response matrices (ARFs) and the detector response matrices (RMFs) were generated by XISRMFGEN and XISARFGEN task pipelines, respectively. The task pipeline ADDASCASPEC was used to combine front-side illuminated spectra and responses. The spectra from XIS0, XIS2, and XIS3 are combined for each observation and referred to in the following as 'front-side illuminated spectra'. The spectrum from XIS1 is referred to in the following as 'back-side illuminated spectrum'. The spectral energy range used in this work is $0.5-10 \mathrm{keV}$.

The data from the hard X-ray detector (HXD) include those from gadolinium silicate crystal counters $\left(\mathrm{Gd}_{2} \mathrm{SiO}_{5}(\mathrm{Ce})\right.$; GSO; $>50 \mathrm{keV})$ and from the silicon PIN diodes $(<50 \mathrm{keV})$. In this work, only PIN data are used, while NGC 5506 was not detected by the GSO. The PIN data was reduced by standard task pipeline HXDPINXBPI. The spectral energy range used in this work is 10 $50 \mathrm{keV}$.

\subsection{NUSTAR}

The data from focal-plane modules (FPM) 1 and 2 were reduced with HEASOFT v6.16, calibration files of INDX20150316. The NUPIPELINE and NUPRODUCTS tasks were run. Source spectra were extracted from a circular region with radius $2^{\prime}$. An off-source background region of the same size was used. The two regions were separated by $1^{\prime}$. The spectral energy range used in this work is 5$79 \mathrm{keV}$.

\footnotetext{
1 It can be found at https://heasarc.gsfc.nasa.gov/docs/suzaku/analysis/abc/.
} 
Table 1. Log of the observations discussed in this paper.

\begin{tabular}{lllll}
\hline \hline Observatory & obsid & $\begin{array}{l}\text { exposure } \\
\text { time [s] }\end{array}$ & $\begin{array}{l}\text { start time } \\
\text { [yyyy-mm-dd hh:mm:ss] }\end{array}$ & $\begin{array}{l}\text { epoch } \\
\text { index }\end{array}$ \\
\hline Chandra & 1598 & 90040 & 2000-12-31 06:21:12 & E01 \\
XMM-Newton & 0013140101 & 20007 & $2001-02-02 ~ 22: 01: 45$ & E02 \\
XMM-Newton & 0201830201 & 21617 & $2004-07-1109: 47: 01$ & E03 \\
XMM-Newton & 0201830301 & 20409 & $2004-07-1422: 11: 39$ & E04 \\
XMM-Newton & 0201830401 & 21956 & $2004-07-2213: 07: 37$ & E05 \\
XMM-Newton & 0201830501 & 20411 & $2004-08-0720: 17: 40$ & E06 \\
Suzaku & 701030010 & 47753 & $2006-08-0816: 30: 05$ & E07 \\
Suzaku & 701030020 & 53296 & $2006-08-1102: 26: 18$ & E08 \\
Suzaku & 701030030 & 57406 & $2007-01-3102: 12: 12$ & E09 \\
XMM-Newton & 0554170101 & 88919 & $2009-01-0219: 32: 26$ & E10 \\
NuSTAR & 60061323002 & 56585 & $2014-04-0123: 41: 07$ & E11 \\
\hline
\end{tabular}

Table 2. Parameters for EPIC data reduction.

\begin{tabular}{lll}
\hline \hline ObsID & $\begin{array}{l}C_{\mathrm{th}}{ }^{a} \\
{\left[\mathrm{~s}^{-1}\right]}\end{array}$ & $\begin{array}{l}R_{\mathrm{s}}{ }^{b} \\
{[\operatorname{arcsec}]}\end{array}$ \\
\hline 0013140101 & $0.2 / 0.5$ & $100 / 40$ \\
0201830201 & $0.5 / 0.5$ & $42 / 45$ \\
0201830301 & $0.5 / 0.35$ & $40 / 42$ \\
0201830401 & $0.35 / 0.35$ & $40 / 42$ \\
0201830501 & $0.35 / 0.35$ & $40 / 42$ \\
0554170101 & $0.35 / 0.35$ & $50 / 50$ \\
\hline${ }^{a}$ Threshold count rate on the high-energy, single-event \\
light curve to identify intervals of high particle \\
background in MOS/PN, respectively. \\
${ }^{b} R_{\mathrm{S}}$ Radius for the source spectrum extraction region.
\end{tabular}

\subsection{Chandra}

The Chandra high energy grating (HEG) and medium energy grating (MEG) spectra in this work were extracted from the Chandra Grating-Data Archive and Catalog (TGCat). Details on the data reduction can be found in Huenemoerder et al. (2011). The spectral energy ranges used in this work are $0.4-5 \mathrm{keV}$ for the two medium energy grating (MEG) spectra, and $0.8-10 \mathrm{keV}$ for the two high energy grating (HEG) spectra.

\subsection{Re-binning spectra}

Spectra were re-binned to ensure that each background-subtracted energy channel had at least one count, and to sample the instrumental energy resolution according to the prescription in Kaastra \& Bleeker (2016).

\subsection{Evolution across 11 epochs}

Fig. 1 illustrates the X-ray spectral variability in NGC 5506 from epoch 1 to 11 . Variability is observed only above $1.3 \mathrm{keV}$. At about $2.5 \mathrm{keV}$, the spectrum change by a factor of 2.5 between the highest and lowest state. These multi-epoch spectra can be classify into three states according to the flux level: high states (A) including E06-E10, intermediate states (B): E01-E05, low state (C): E11. NGC 5506 evolved from intermediate states (E01-E05), to high states (E06-E10), and reached finally a low state (E11).

\section{SPECTRAL RESULTS}

The spectral fits are performed using XSPEC v.12.8.2 (Arnaud 1996). The statistical uncertainties reported in this paper corresponds to $90 \%$ confidence level for one interesting parameter.

\subsection{The soft X-ray spectrum}

In order to achieve a broad-band modeling of the NGC5506 X-ray spectra, we considered initially the soft X-ray band $(E<1.3 \mathrm{keV})$ only. Our working hypothesis is that soft $\mathrm{X}$-rays are dominated by emission due to diffuse gas associated to extended Narrow Line Regions, while emission at higher energy is dominated by the nuclear emission. Different models of the soft X-ray spectrum are compared in Tab. 3. Comparing models of increasing level of complexity via the F-test, we define the best-fit baseline model of the soft X-ray spectrum as: POWERLAW[1] + XSTAR2XSPEC + APEC[1] + APEC[2] to model the soft X-ray component. Each component is described in the following.

- XSTAR2XSPEC (NGC5506) is an additive table model generated by XSTAR. XSTAR (Kallman \& Bautista 2001) calculates the physical conditions and emission spectra of photoionized gases. It is parameterized by a column density $N_{\mathrm{H}}^{\text {phot. }}$ and ionisation $\xi_{\text {phot. }}$. PEGPWRLW is a power-law component (with photon in$\operatorname{dex} \alpha_{\text {phot. }}$ ) approximating free electron scattering in the photoionized gas. APEC (Smith et al. 2001) components are emission spectra from collisionally-ionized diffuse gases of temperatures $k T_{1}^{\text {coll. }}$ and $k T_{2}^{\text {coll. }}$ calculated using the ATOMDB code v2.0.2. ${ }^{2}$. All parameters mentioned in this paragraph are left epoch-invariant. The best-fit parameters are shown in Tab. 5 .

To test if the soft X-ray emission is constant during our observations (as expected on astrophysical grounds; see Sect. 1), RGS spectra of each epoch were fit using the aforementioned model. Constant-functions were fit to soft X-ray parameters as a function of time, and then the probability of the validity of this assumption was calculated. The results of this test are reported in Tab. 4. The high $p$-values of fits suggest that invariant model parameters describe the soft RGS spectra properly. To reduce the statistical uncertainties in RGS measurements, spectra from different epochs were merged. Hereafter, the parameters of the model describing the soft X-ray band are assumed to be epoch-independent.

2 http://atomdb.org/ 


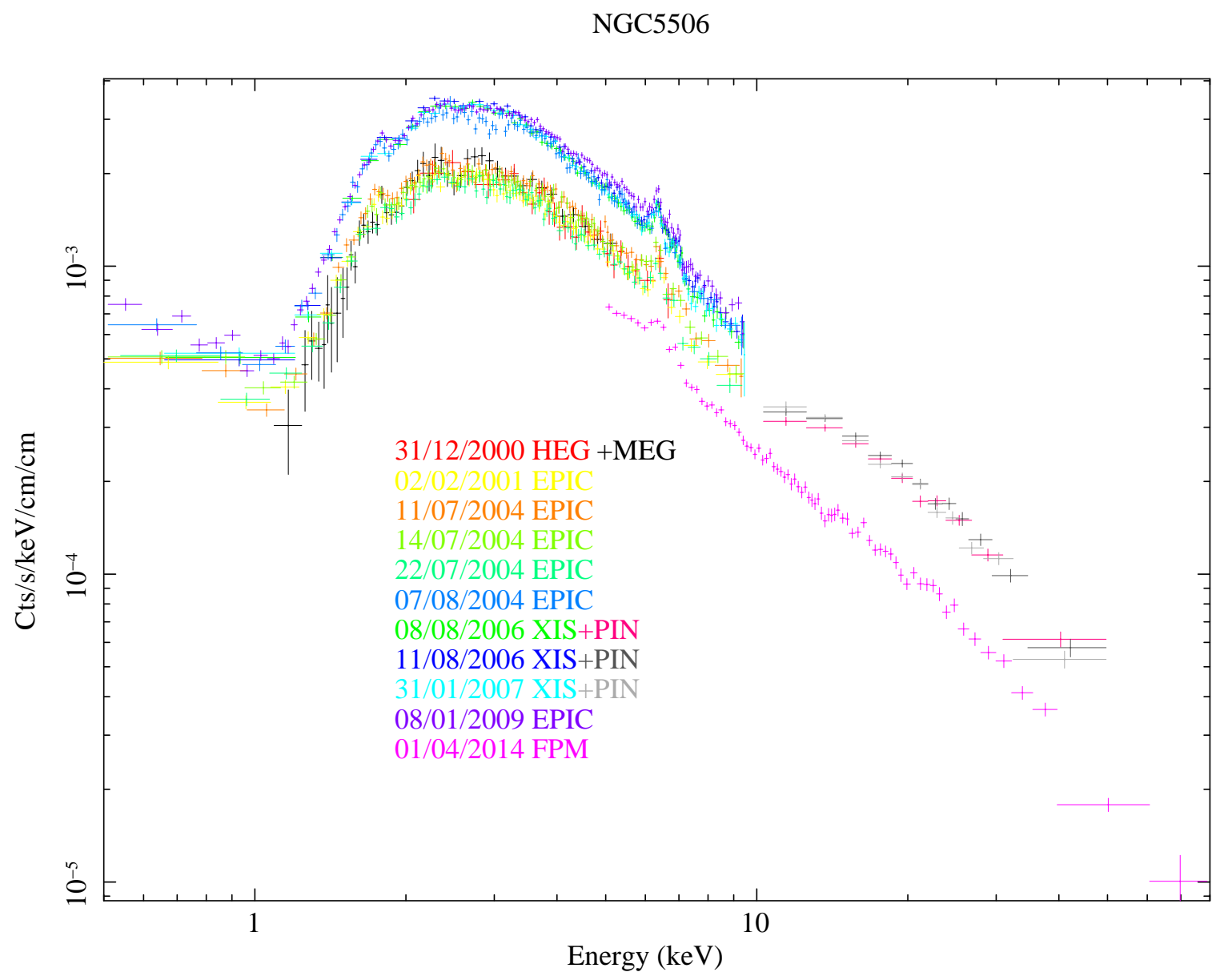

Figure 1. NGC 5506 count spectra from Chandra, XMM-Newton, Suzaku, and NuSTAR (corrected by effective areas). The observation dates, instruments, and corresponding colors are labeled in the figure. The intrinsic variability of the source at energies $>2 \mathrm{keV}$ can be directly seen. For example, at $3 \mathrm{keV}$ EPIC spectra from 08/01/2009 and 14/07/2004 are different by a factor of $\sim 2$.

Table 3. Models for high-resolution spectra below $1.3 \mathrm{keV}$.

\begin{tabular}{lrrc}
\hline \hline model & C-Statistic & $\chi^{2}$ n.d.f. & F-test prob. ${ }^{\mathbf{2}}$ \\
\hline $\mathrm{A}($ nucl.) & 18593 & $16847 / 5496=3.065$ & - \\
B(nucl. + coll.) & 6771 & $6412 / 5493=1.167$ & $4.0 \times 10^{-17}[\mathrm{~A}]$ \\
C(nucl. + phot.) & 6356 & $6185 / 5491=1.126$ & $9.5 \times 10^{-11}[\mathrm{~A}]$ \\
D(nucl. + phot. + coll.) & 6186 & $6071 / 5488=1.106$ & $3.6 \times 10^{-8}[\mathrm{C}]$ \\
E(nucl. + phot. + 2 coll.) & 6160 & $6030 / 5485=1.099$ & $2.2 \times 10^{-7}[\mathrm{D}]$ \\
\hline
\end{tabular}

A: $\operatorname{tBABS}[1] *(\operatorname{tBABS}[2] *$ POWERLAW[2])

B: $\operatorname{tBABS}[1] *($ APEC + tBABS[2]*POWERLAw[2] $)$

C: $\operatorname{tBABS}[1] *($ POWERLAW[1] + XSTAR2XSPEC + TBABS[2]*POWERLAW[2])

D: $\operatorname{TBABS}[1] *($ POWERLAW $[1]+$ XSTAR2XSPEC + APEC + TBABS[2]*POWERLAW[2] $)$

E: tBabs[1] $*$ (POWERLAW[1] + XSTAR2XSPEC + APEC[1] + APEC[2] + TBABS[2]*POWERLAW[2])

See description for each model in Subsect. 3.1.

number of PHA bins: 5500

* the probability that the hypothesized model is worse; comparing with the null model in [ ]

\subsection{Broadband X-ray modeling}

We then fit the X-ray broadband spectra of the 11 epochs with the following model:
Table 4. Constant-function fitting to RGS epoch-dependent parameters

\begin{tabular}{lll}
\hline \hline parameter & $\chi^{2}$ n.d.f. & p-value \\
\hline$\alpha_{\text {phot. }}$ & $4.07 / 5$ & 0.539 \\
$k T_{1}^{\text {coll. }}$ & $3.64 / 5$ & 0.603 \\
$Z$ & $0.65 / 5$ & 0.986 \\
$k T_{2}^{\text {coll. }}$ & $0.50 / 5$ & 0.992 \\
$\log \xi_{\text {phot. }}$ & $4.97 / 5$ & 0.420 \\
\hline
\end{tabular}

number of entries: 6 (epochs)

number of parameter: 1 (constant-function)

See description for each parameter in Subsect. 3.2.

XSTAR2XSPEC, PEGPWRLW, and APEC, are explained in Sect. 3. The other components are described in the following.

- Mytorus ${ }^{3}$ (Murphy \& Yaqoob 2009) was used to calculate the reflection and scattering from optically thick matter in a toroidal geometry surrounding the AGN. In this work, this torus model set includes $\mathrm{MT}_{\mathrm{sca}}$ and $\mathrm{MT}_{\text {lin. }}$. $\mathrm{MT}_{\mathrm{sca}}$ calculates the scattered continuum. It is the collection of all escaping photons that have been scattered in the medium at least once. It is also referred to as the

3 http://mytorus.com/

CONST $*$ TBABS $1 *$ PEGPWRLW + APEC $1+$ APEC $2+$ XSTAR 2 XSPEC

$+\mathrm{TBABS} *$ RELXILL $+\mathrm{MT}_{\mathrm{sca}}+\mathrm{MT}_{\text {lin }}$ 
reflection spectrum. $\mathrm{MT}_{\text {lin }}$ calculates the fluorescent emission-line spectrum, which is produced by the zeroth-order (or unscattered) component resulting from the absorption of a continuum photon above the K-edge threshold as well as by the emission-line photons that are scattered before they escape the medium. It includes the Compton shoulder of the observed emission line. The column density of the reprocessing matter $N_{\mathrm{H}}^{\text {torus }}$, the inclination angle $\theta_{\text {torus }}$, and the photon index of the reprocessed primary continuum $\alpha_{\text {torus }}$, are assumed epoch-invariant, following Guainazzi et al. (2010a).

- RELXILL (Dauser et al. 2014; García et al. 2014) describes an $\mathrm{X}$-ray source of a power-law photon index $\alpha_{\text {coro. }}$ and a cutoff at high energies $E_{\text {cut }}^{\text {coro. }}$ plus the X-ray reflection from a relativistically rotating disk in a strong gravitational field generated by a (possibly spinning) black hole. We assumed that the emission occurs in an annulus over the accretion disk, whose outer radius was fixed to $400 r_{\mathrm{g}}$, and whose inner radius coincides with the innermost stable circular orbit (ISCO), which is a function of the BH spin $a$ (Bardeen et al. 1972). We assumed that the radial profile of the emissivity function can be described by a single power-law of in$\operatorname{dex} \beta_{\mathrm{AD}}$. The inclination angle of the $\mathrm{AD}$ is $\theta_{\mathrm{AD}}$. The definition of parameter reflection fraction $F_{\text {refl. }}$ can be found in (Dauser et al. 2014). The following astrophysical parameters: $\alpha_{\text {coro. }}, \beta_{\mathrm{AD}}$, ionisation parameter of AD $\xi_{\mathrm{AD}}$, and normalisation of RELXILL $N_{0}^{\mathrm{RELX}}$. are assumed independently variable in each epoch, while the other parameters are left epoch-invariant.

- CONST represents the cross-instrument calibration factors. TBABS[2] describes the obscuration of the nuclear emission by a hydrogen column density $N_{\mathrm{H}}^{\text {nucl. }}$. TBABS[1] describes obscuration due to intervening gas in our Galaxy by a hydrogen column density $N_{\mathrm{H}}^{\mathrm{gala}}$. All parameters mentioned in this paragraph are left epochinvariant except $N_{\mathrm{H}}^{\text {nucl. }}$.

- We assumed a variable and epoch-invariant elemental abundance $Z$. Its value is assumed to be consistent across models (APEC[1], APEC[2], and RELXILL).

\subsection{1 fit results}

Spectra are shown epoch by epoch in the upper panels of Figs. 2 to 3 , and their data/model ratios in the lower panels.

The the best-fit results of stable (epoch-invariant) astrophysical properties are listed in Tab. 5. The parameters that vary with epochs and drive the evolution of the spectrum are in Tab. 6 .

\subsection{2 nuclear emission}

In this paper, we employed the state-of-the-art RELXILL model to model the reflection spectrum due to the X-ray illuminated relativistic accretion disk. This allows us to obtain statistical constraints on: a $\mathrm{BH}$ of spin $0.97 \pm_{0.010}^{0.0082}$ with an $\mathrm{AD}$ inclination angle $41_{-0.18}^{\circ+0.12}$, emissivity indices varying from 1.2 to 8.5 , ionisation parameter $\log \left(\xi_{\mathrm{AD}}\right)$ varying in the range 1.6 to above 4.7 , a corona of photo-index varying between 1.7-2.1, and a high-energy cutoff $\sim 500 \mathrm{keV}$. The reflection fraction is $\sim 1.3$ (implying the corona residing $\sim 20 r_{\mathrm{g}}$ above the black hole ${ }^{4}$ ).

We compare our results with those in other studies: in the paper of Matt et al. (2015) (NGC 5506 by NuSTAR), the AD inclination angle was reported as $<44^{\circ}$, consistent to what derived in this work; ionisation $\xi=22_{-7}^{+15}$, which is smaller than that for

4 converted through the plot of "reflection fraction v.s. height of corona" in Dauser et al. (2016).
Table 5. Fit results of epoch-independent parameters.

\begin{tabular}{|c|c|c|c|}
\hline $\begin{array}{l}\text { Par. } \\
\text { name }\end{array}$ & $\begin{array}{l}\text { Par. } \\
\text { unit }\end{array}$ & $\begin{array}{l}\text { best-fit } \\
\text { value }\end{array}$ & \\
\hline$N_{\mathrm{H}}^{\text {gala. }}$ & $\mathrm{cm}^{-2}$ & $3.6 \pm_{0.072}^{0.10}$ & $\times 10^{-21}$ \\
\hline$\alpha_{\text {phot. }}$ & & $2.8 \pm_{0.16}^{0.13}$ & \\
\hline$k T_{1}^{\text {coll. }}$ & $\mathrm{keV}$ & $6 \pm_{0.7}^{0.2}$ & $\times 10^{-2}$ \\
\hline Z & solar abundance & $5.4 \pm_{0.10}^{0.15}$ & $\times 10^{-1}$ \\
\hline$k T_{2}^{\text {coll. }}$ & $\mathrm{keV}$ & $3.0 \pm_{0.14}^{0.13}$ & $\times 10^{-1}$ \\
\hline $\log \xi_{\text {phot. }}$ & & $1.8 \pm_{0.16}^{0.10}$ & \\
\hline$a$ & & $9.7 \pm_{0.10}^{0.082}$ & $\times 10^{-1}$ \\
\hline$\theta_{\mathrm{AD}}$ & deg & $4.1 \pm_{0.018}^{0.012}$ & $\times 10^{1}$ \\
\hline$E_{\text {cut }}^{\text {coro. }}$ & $\mathrm{keV}$ & $5.0 \pm_{0.13}^{0.10}$ & $\times 10^{2}$ \\
\hline$F_{\text {refl. }}$ & & $1.3 \pm_{0.024}^{0.010}$ & \\
\hline$N_{\mathrm{H}}^{\text {torus }}$ & $\mathrm{cm}^{-2}$ & $5 \pm_{0.3}^{0.1}$ & $\times 10^{24}$ \\
\hline$\theta_{\text {torus }}$ & $\operatorname{deg}$ & $4.7 \pm_{0.19}^{0.10}$ & $\times 10^{1}$ \\
\hline$\alpha_{\text {torus }}$ & & $2.0 \pm_{0.015}^{0.010}$ & \\
\hline$E_{1}^{\text {line }}$ & $\mathrm{keV}$ & $1.7 \pm_{0.072}^{0.023}$ & \\
\hline$\sigma_{1}^{\text {line }}$ & $\mathrm{keV}$ & $1 \pm_{0.2}^{0.1}$ & $\times 10^{-1}$ \\
\hline$E_{2}^{\text {line }}$ & $\mathrm{keV}$ & $6.7 \pm_{0.10}^{0.18}$ & \\
\hline$\sigma_{2}^{\text {line }}$ & $\mathrm{keV}$ & $2.2 \pm_{0.042}^{0.050}$ & $\times 10^{-1}$ \\
\hline
\end{tabular}

E11 in this work $(\log \xi \sim 4.5)$; corona photo-index 1.9 , which is harder than that for E11 in this work; a very high-energy cutoff at $720_{-190}^{+130} \mathrm{keV}$, which is higher than in this work, but still consistent with our results.

The radial index of the emissivity profile exhibits large errors. A test of making this parameter epoch-independent was performed. There was no great change in data-model residuals of each spectrum. The value of C-Statistic increased by 199, while the number of parameters decreased by 11. C-Statistic/d.o.f: 12443/10154 $=1.225$ (original); $12642 / 10165=1.244$ (this test). A F-test was made to compare these two hypotheses. The restricted hypothesis (the index epoch-invariant) has a Chi-Squared of 11662 and a number of free parameters 99 (d.o.f 10165) while the unrestricted hypothesis (epoch-variant) has a Chi-Squared of 11498 and a number of free parameters 110 (d.o.f 10154). We found that the hypothesis of epoch-dependent $\beta_{\mathrm{AD}}$ is better with a confidence level of more than $99 \%$.

Suzaku/XIS spectra show positive residuals at $\sim 7 \mathrm{keV}$ (see an example of this feature in Fig. 4). This might be the signature of emission from resonant $\mathrm{H}$-like iron lines. Therefore, we added a further, unresolved Gaussian profile to the model, constraining its rest-frame centroid energy to be comprised between 6.6 and $7 \mathrm{keV}$. The value of goodness-of-fit (C-Statistic) decreased by 190, while the number of free parameters increased by 3. C-Statistic/d.o.f: 
RGS

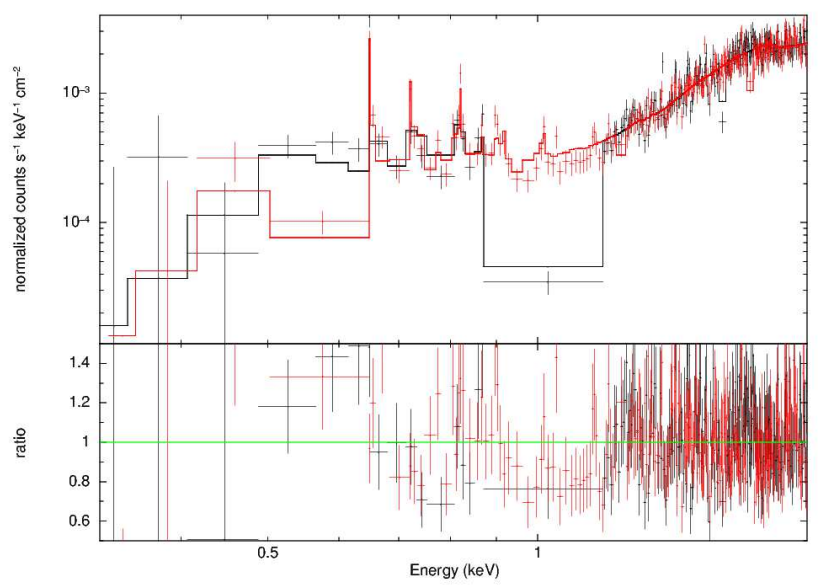

02/02/2001 EPIC

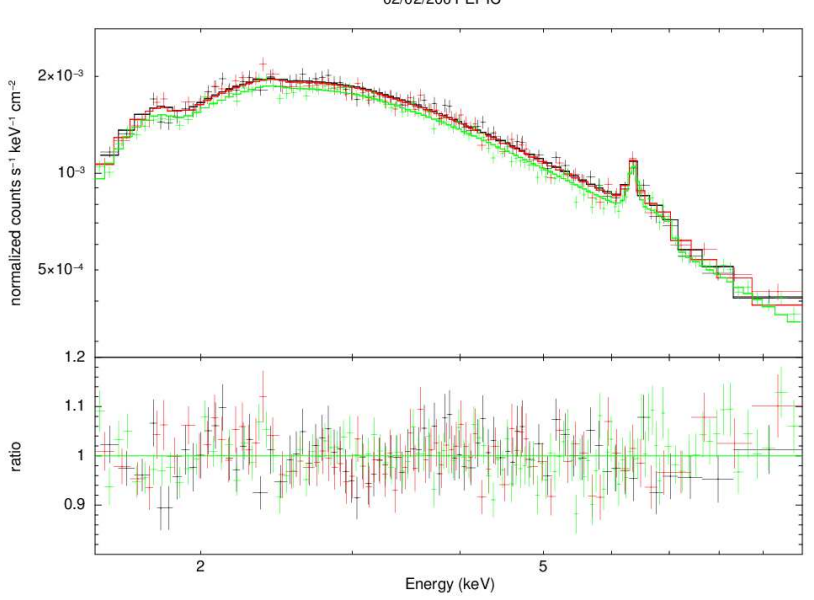

14/07/2004 EPIC

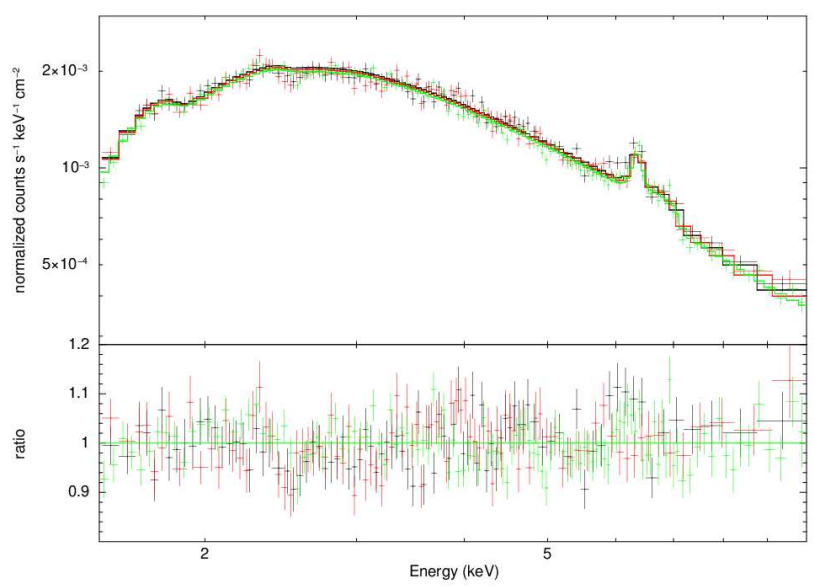

31/12/2000 HETG

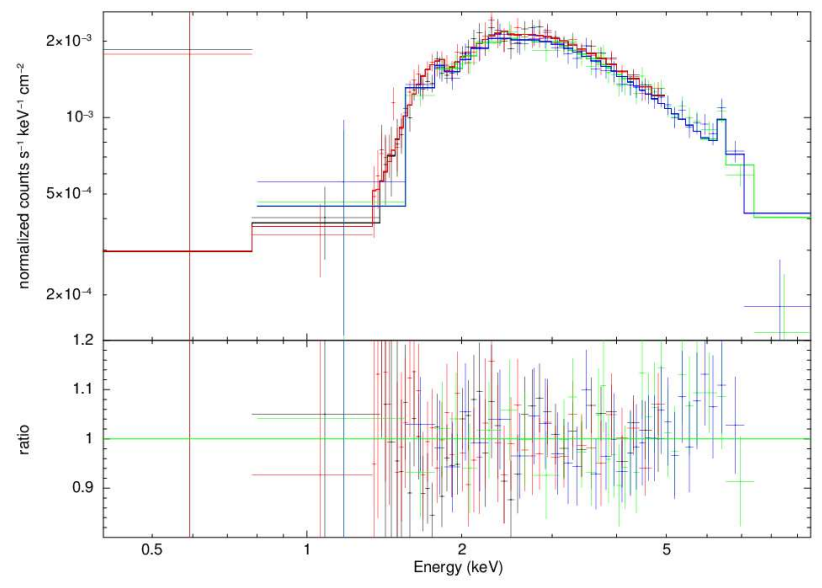

11/07/2004 EPIC

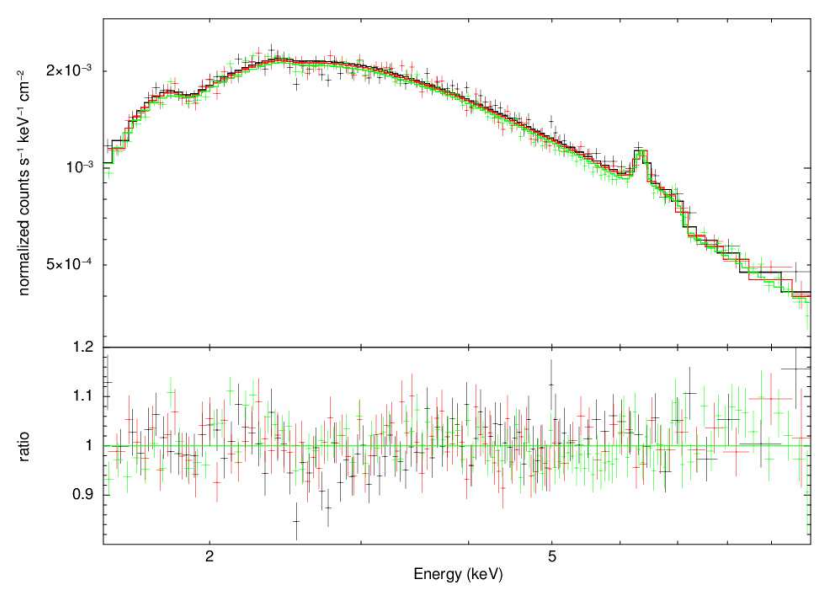

22/07/2004 EPIC

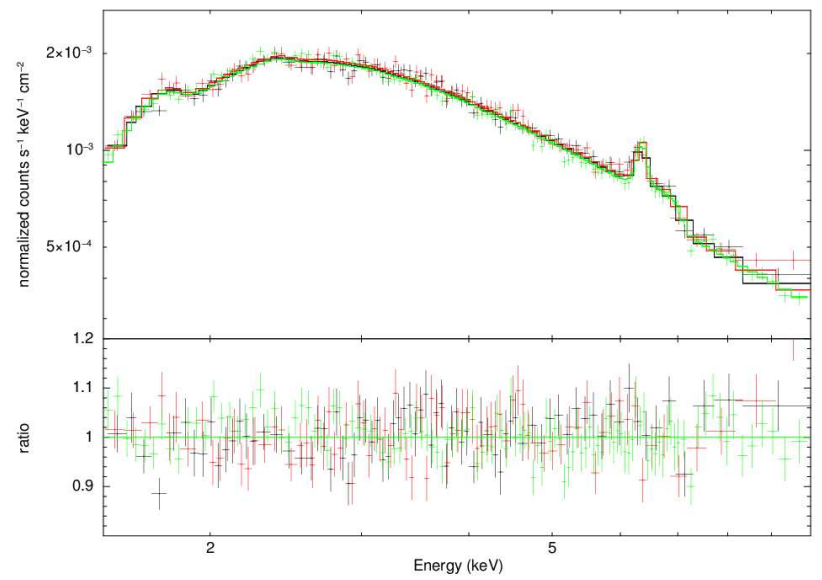

Figure 2. Upper panels: NGC 5506 X-ray spectra from RGS and Epochs 1-5, and their best-fit models. The instrument name and observation date for each epoch are on the top the panel. Crosses: data; histogram lines: models. Lower panels: the data/model ratios.

$12443 / 10154=1.225$ (original); $12253 / 10151=1.207$ (this test, adding a Gaussian profile). A F-test was made to compare these two hypotheses. The restricted hypothesis (without a Gaussian profile) has a Chi-Squared of 11498 and a number of free parameters 110 (d.o.f 10154) while the unrestricted hypothesis (with a Gaussian profile) has a Chi-Squared of 11336 and a number of free parame- ters 113 (d.o.f 10151). We found that the model with this Gaussian profile is better at a confidence level of larger than $99 \%$.

In Figs. 5 and 6, we show the NUSTAR residuals once the normalisation of the RELXILL reflection component is set to zero, to show the effect that the relativistically smeared accretion disk reflection component has on the final best-fit model. 

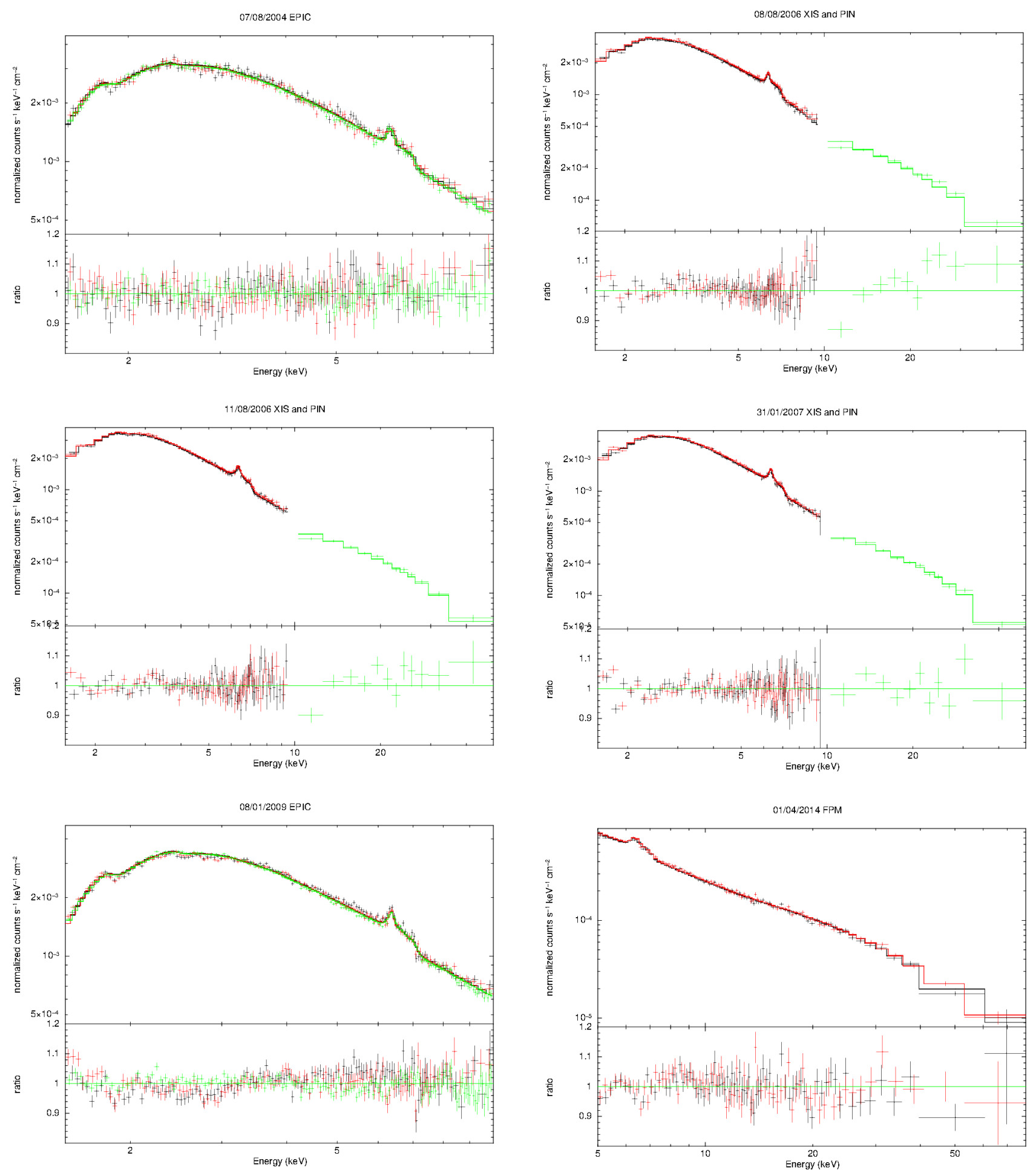

Figure 3. NGC 5506 X-ray spectra from Epochs 6-11, and their best-fit models. See description in the caption of Fig.2.

A Gaussian profile at about $1.7 \mathrm{keV}$ also is required by the fit. We do not have an explanation for this feature that we tentatively attribute to calibration uncertainties at the energies of the Si escape peak..

\subsection{3 obscuration and torus}

The column density of the nuclear absorber varies in the range $2.8-$ $3.4 \times 10^{22} \mathrm{~cm}^{-2}$. Matt et al. (2015) reported $3.1_{-0.20}^{+0.21} \times 10^{22} \mathrm{~cm}^{-2}$, which agrees with the result in this work. Ginga also observed variation in the column density in a timescale of $\sim 1-\sim 10$ days in 1991 (Bond et al. 1993). This timescale is comparable with that presented in this paper, which corresponds to the coherent change 
Table 6. Best-fit values of the time-dependent parameters.

\begin{tabular}{ccccccc}
\hline \hline epoch & $\begin{array}{c}\text { start time } \\
{[\mathrm{MJD}]}\end{array}$ & $\begin{array}{c}\text { energy flux }(2-10 \mathrm{keV})^{4} \\
{\left[10^{-11} \mathrm{ergs}^{-1} \mathrm{~cm}^{-2}\right]}\end{array}$ & $\begin{array}{c}N_{\mathrm{H}}^{\text {nucl. }} \\
{\left[10^{22} \mathrm{~cm}^{-2}\right]}\end{array}$ & $\beta_{\mathrm{AD}}$ & $\alpha_{\text {coro. }}$ & $\log \xi_{\mathrm{AD}}$ \\
\hline E01 & 51909 & $6.0 \pm_{0.041}^{0.042}$ & $3.3 \pm_{0.017}^{0.017}$ & $5.0 \pm_{0.81}^{0.89}$ & $2.0 \pm_{0.051}^{0.010}$ & $4.7 \pm_{0.13}^{0.15}$ \\
E02 & 51942 & $6.2 \pm_{0.030}^{0.030}$ & $2.8 \pm_{0.031}^{0.023}$ & $8.5 \pm_{0.32}^{0.11}$ & $1.7 \pm_{0.042}^{0.010}$ & $3.6 \pm_{0.14}^{0.48}$ \\
E03 & 53197 & $6.9 \pm_{0.030}^{0.030}$ & $3.0 \pm_{0.056}^{0.013}$ & $3.3 \pm_{0.45}^{0.53}$ & $1.8 \pm_{0.057}^{0.010}$ & $4.0 \pm_{0.13}^{0.67}$ \\
E04 & 53200 & $6.7 \pm_{0.030}^{0.031}$ & $3.0 \pm_{0.011}^{0.024}$ & $6.5 \pm_{0.81}^{0.89}$ & $1.7 \pm_{0.010}^{0.019}$ & $3.5 \pm_{0.11}^{0.10}$ \\
E05 & 53208 & $6.1 \pm_{0.029}^{0.029}$ & $3.2 \pm_{0.024}^{0.021}$ & $4.0 \pm_{0.56}^{0.95}$ & $1.9 \pm_{0.010}^{0.041}$ & $4.0 \pm_{0.15}^{0.10}$ \\
E06 & 53234 & $9.7 \pm_{0.036}^{0.036}$ & $2.8 \pm_{0.058}^{0.010}$ & $4.0 \pm_{0.33}^{0.36}$ & $1.8 \pm_{0.028}^{0.0082}$ & $3.5 \pm_{0.12}^{0.11}$ \\
E07 & 53955 & $9.8 \pm_{0.028}^{0.028}$ & $3.1 \pm_{0.010}^{0.011}$ & $2.0 \pm_{0.21}^{0.28}$ & $2.0 \pm_{0.025}^{0.010}$ & $1.6 \pm_{0.26}^{0.12}$ \\
E08 & 53958 & $10 \pm_{0.027}^{0.027}$ & $3.1 \pm_{0.018}^{0.010}$ & $1.2 \pm_{0.57}^{0.48}$ & $1.7 \pm_{0.082}^{0.11}$ & $3.0 \pm_{0.19}^{0.14}$ \\
E09 & 54131 & $9.9 \pm_{0.025}^{0.025}$ & $3.2 \pm_{0.010}^{0.012}$ & $1.6 \pm_{0.71}^{0.47}$ & $2.1 \pm_{0.010}^{0.048}$ & $3.8 \pm_{0.21}^{0.22}$ \\
E10 & 54833 & $11 \pm_{0.021}^{0.021}$ & $3.1 \pm_{0.010}^{0.060}$ & $2.6 \pm_{0.078}^{0.050}$ & $1.9 \pm_{0.010}^{0.043}$ & $3.2 \pm_{0.13}^{0.14}$ \\
E11 & 56748 & $5.8 \pm_{0.021}^{0.022}$ & $3.4 \pm_{0.071}^{0.043}$ & $3.5 \pm_{0.062}^{0.033}$ & $2.1 \pm_{0.068}^{0.010}$ & $4.5 \pm_{0.15}^{0.12}$ \\
\hline
\end{tabular}

See description for each parameter in Subsect. 3.2.

- energy flux 2-10 keV calculated with spectrum by CFLUX in XSPEC

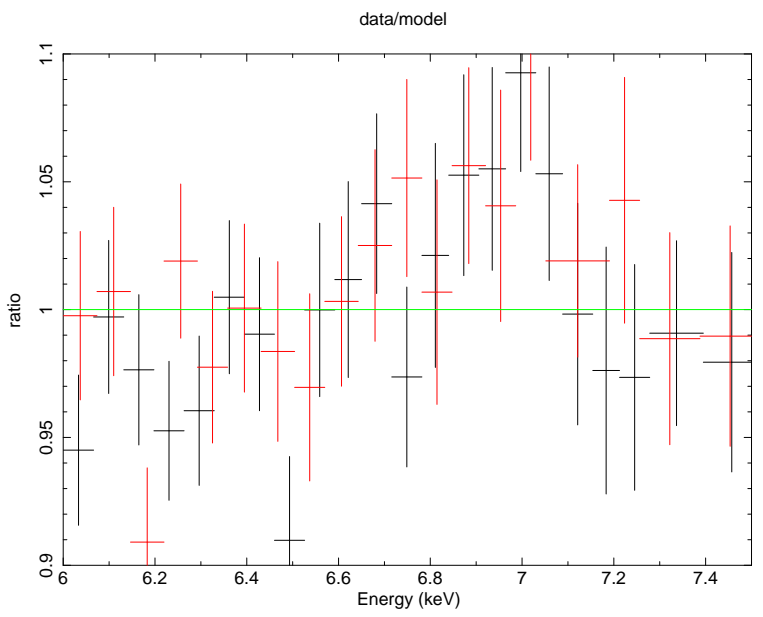

Figure 4. Data/model ratio when the baseline model is applied to the XIS spectra of Epoch E08 in the 6-7.5 keV energy range.

timescale of an astrophysical structure of $\sim 0.001-\sim 0.01 \mathrm{pc}$. This size is similar to that of a broad-line region (BLR) of an $\mathrm{AGN}^{5}$.

The best-fit inclination angle of the torus is about $47^{\circ}$. The matter in the torus does not intercept the line of sight, and reflects the nuclear emission. The column density of the torus is $\sim 5 \times$ $10^{24} \mathrm{~cm}^{-2}$. Note that this time-invariant column density is different from that on the LOS, $N_{\mathrm{H}}^{\text {nucl. }}$.

Model TBABS[1] represents the medium with stable column density $\left(3.6 \times 10^{21} \mathrm{~cm}^{-2}\right)$ on the LOS from the outer soft-excess

5 A typical size of BLR of an AGN is $10^{4}-10^{5} r_{\mathrm{g}}$ (Netzer 2013); $r_{\mathrm{g}}=$ $G M c^{2}$. In the case of $\mathrm{NGC} 5506, \log \left(M_{\mathrm{BH}} / \mathrm{M}_{\odot}\right)=7.95$ (Marinucci et al. 2012).

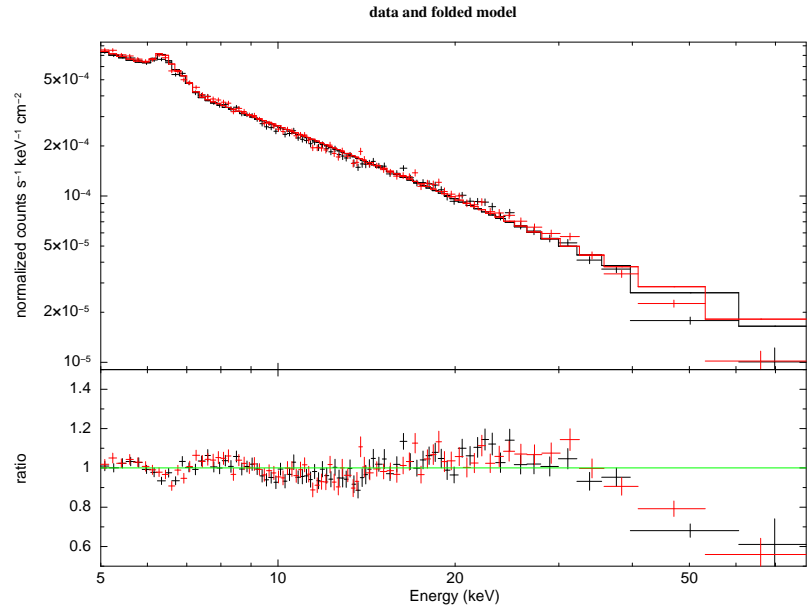

Figure 5. The FPM spectra and the best-fit model without the relativistic disk reflection component.

emitting region to the observer, including the medium causing the galactic extinction, inter-galactic medium, and galactic medium in NGC 5506.

\subsection{Correlation results}

Spearman's ranking correlation coefficients between several pairs of parameters were calculated and reported in Tab. 7. It is found that $\beta_{\mathrm{AD}}, \log \xi_{\mathrm{AD}}, \alpha_{\text {coro. }}, N_{\mathrm{H}}^{\text {nucl. }}$ are all anti-correlated with the energy flux. The correlation between the column density and the photoindex is a degeneracy in the fit.

The fitting procedure employs C-Statistic (Cash 1979). The goodness-of-fit is 12260 for 10158 degrees of freedom. 


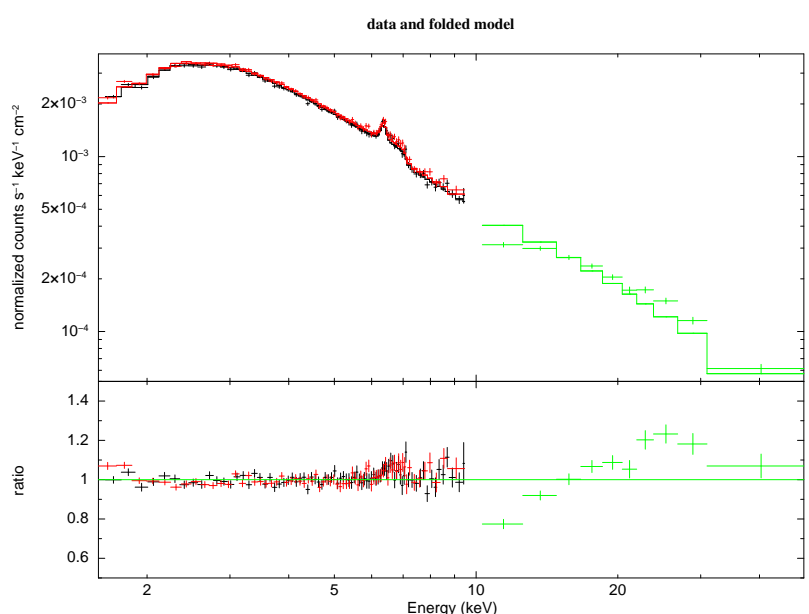

Figure 6. The XIS and PIN spectra from Epoch 7, and the best-fit model without the relativistic disk reflection component.

Table 7. Spearman's ranking correlation coefficients between pairs of epoch-dependent parameters.

\begin{tabular}{lllc}
\hline \hline $\mathrm{X}$ & $\mathrm{Y}$ & $\rho$ & $\begin{array}{c}\text { two-tailed } \\
\text { probability } \\
P \text {-value }\end{array}$ \\
\hline energy flux & $N_{\mathrm{H}}^{\text {nucl. }}$ & -0.314 & 0.347 \\
energy flux $^{\star}$ & $\alpha_{\text {coro. }}$ & -0.171 & 0.614 \\
energy flux & $\beta_{\mathrm{AD}}$ & -0.702 & 0.016 \\
energy flux & $\log \xi_{\mathrm{AD}}$ & -0.781 & 0.005 \\
$N_{\mathrm{H}}^{\text {nucl. }}$ & $\alpha_{\text {coro. }}$ & 0.786 & 0.004 \\
$N_{\mathrm{H}}^{\text {nucl. }}$ & $\log \xi_{\mathrm{AD}}$ & 0.492 & 0.124 \\
$N_{\mathrm{H}}^{\text {nucl. }}$ & $\beta_{\mathrm{AD}}$ & -0.287 & 0.392 \\
$\beta_{\mathrm{AD}}$ & $\log \xi_{\mathrm{AD}}$ & 0.412 & 0.208 \\
$\log \xi_{\mathrm{AD}}$ & $\alpha_{\text {coro. }}$ & 0.398 & 0.226 \\
$\beta_{\mathrm{AD}}$ & $\alpha_{\text {coro. }}$ & -0.325 & 0.330 \\
\hline
\end{tabular}

- energy flux 2-10 keV calculated with spectrum by CFLUX in XSPEC

\section{DISCUSSION AND CONCLUSIONS}

In this paper, all the good quality X-ray archival data of NGC 5506 are analyzed simultaneously to obtain the unprecedented combination of high signal-to-noise ratio and broadband energy coverage for the precise measurement of the black hole spin. There have been several spin measurements for the SMBHs of AGNs through characterizing the relativistic reflection spectra in X-ray (Walton et al. 2013; Tan et al. 2012; Lohfink et al. 2012; Zoghbi et al. 2010). For NGC 5506, the SMBH spin had not been measured. In a previous study on NGC 5506 (Guainazzi et al. 2010b), the data quality (on a smaller data set than discussed in this paper) did not allow to constrain the spin: varying the black hole spin from Schwarzschild to maximally rotating yields a variation of the $\chi^{2}$ lower than 0.5 . In this paper, we constrain the black hole spin to $a=0.97 \pm_{0.010}^{0.0082}$ at $90 \%$ confidence level for one interesting parameter. There have been several measurements where uncertainties in the spin parameter were estimated by 1-D confidence contours, reporting uncertainty ranges of $90 \%$ confidence between 0.01 and 1 (e.g. Reynolds (2014)); in this paper, the uncertainty range is 0.018 . In Fig. 7, the SMBH mass-spin distribution of NGC 5506 with that of other AGNs collected in Reynolds (2014) are compared. There is a trend, whereby smaller black hole masses correspond to higher black hole

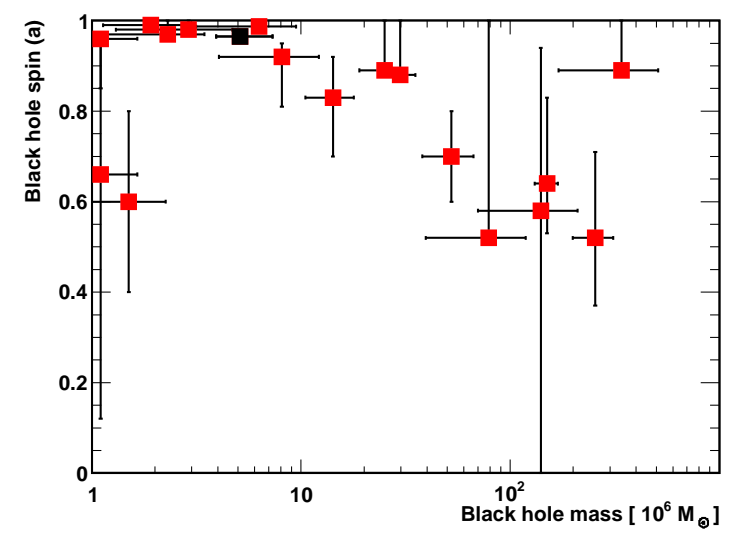

Figure 7. Black hole mass/spin measurements of NGC 5506 (in black; Nikołajuk et al. (2009)/this paper) and other AGNs (in red; data collected in Reynolds (2014).

Table 8. Constant-function fitting to epoch-dependent parameters.

\begin{tabular}{lll}
\hline \hline parameter & $\chi^{2}$ n.d.f. & p-value \\
\hline$N_{\mathrm{H}}^{\text {nucl. }}$ & $\left(2.46 \times 10^{3}\right) / 11$ & 0.00 \\
$\beta_{\mathrm{AD}}$ & $\left(8.15 \times 10^{1}\right) / 11$ & $2.59 \times 10^{-13}$ \\
$\alpha_{\mathrm{coro} .}$ & $\left(4.93 \times 10^{1}\right) / 11$ & $3.62 \times 10^{-7}$ \\
$\log \xi_{\mathrm{AD}}$ & $\left(2.61 \times 10^{3}\right) / 11$ & 0.00 \\
\hline number of entries: 11 (epochs) \\
number of parameter: 1 (constant-function) \\
\multicolumn{2}{l}{ See description for each parameter in Subsect. 3.2. }
\end{tabular}

spin. While the statistics is still small, NGC5506 agrees with this trend.

In our model, five parameters are epoch-dependent: $N_{\mathrm{H}}^{\text {nucl. }}$, $\beta_{\mathrm{AD}}, \log \xi_{\mathrm{AD}}, \alpha_{\mathrm{coro}}$, and $N_{0}^{\mathrm{RELX}}$. They are all variable over time scales probed by our data (see Tab. 6).

In the correlation study (see Tab. 7), there are three correlations significant at a level better than $2 \%$. The $N_{\mathrm{H}}^{\text {nucl. }}$ versus $\alpha_{\text {coro. }}$ is an intrinsic degeneracy between these parameters in the fit. The flux versus index of the radial emissivity profile, and flux versus ionization parameter anti-correlations may suggest that something in the accretion flow changes with flux. A working hypothesis is that what changes is the location of the innermost stable orbit due to over-ionisation of the innermost regions of the disk. At higher fluxes, a wider area of the innermost disk is fully ionized and does not contribute to the reflection. If this happens, the relative weight of disk regions increases that correspond a lower index of the radial emissivity profile and to a lower ionization level (i.e., ionization parameter). Hence it came the idea of changing slightly the baseline model, assuming a constant index of the radial emissivity profile (3), and allowing the radius of the innermost stable orbit $\left(r_{\text {in }}\right)$ to vary at different epochs (modified model hereafter). If this working hypothesis is correct, we should see a positive correlation between flux and $r_{\text {in }}$, and this is indeed what the modified model shows. See Fig. 8. The resulted $r_{\text {in }}$ has a Spearman's correlation coefficient 0.754 with energy flux (a confidence level of 99\%). While formally there is a statistical linear correlation between energy flux and $\log r_{\text {in }}$, at high flux levels $\left(>9 \times 10^{11} \mathrm{cgs}\right)$ two out of five data points are consistent to those measured at low flux levels. More than a linear correlation, there seems to be a special "high flux state" $\left(r_{\text {in }}>10 r_{\mathrm{g}}\right)$ where the properties of the accretion flow differ from 


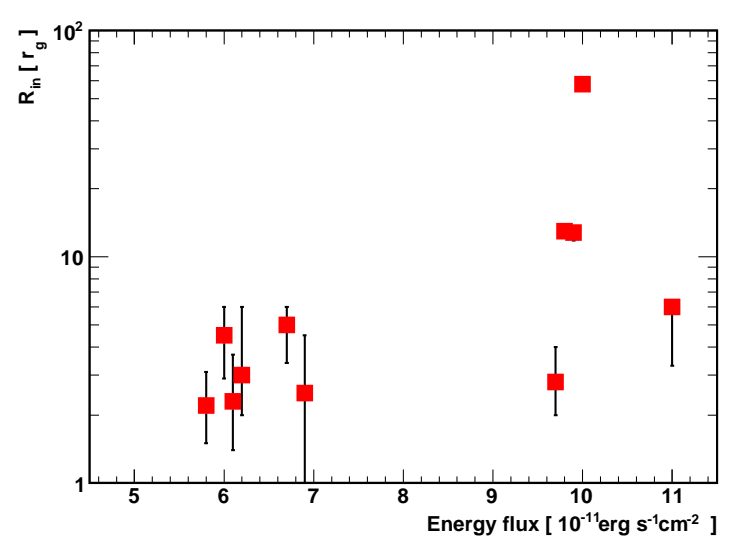

Figure 8. Correlation between energy flux and $r_{\text {in }}$ in the modified model (see the description in Sect. 4).

any other flux state. These three data points with $r_{\text {in }}>10 r_{\mathrm{g}}$ correspond to epochs 7, 8, and 9 when Suzaku observed NGC 5506.

In this modified model, our spectral modeling is inconsistent: the measurement of the black hole spin assumes that the disk reflection is produced down to the ISCO at all epochs. Therefore, we checked its impact on the determination of the spin: $a=0.93 \pm_{0.016}^{0.0063}$ (in the modified model) at $90 \%$ confidence level for one interesting parameter, which is slightly lower than $a=0.97 \pm_{0.010}^{0.0082}$ in the baseline model. We can see that the statistical accuracy in the measurement of the black hole spin achieved by our study is already excellent, but the total uncertainty is larger as reflected in modified model. This uncertainty (i.e., our degree of ignorance of the black hole spin in NGC 5506) is dominated by our ignorance in the physics of the underlying accretion flow, which we have only superficially touched through the modified model. Therefore, the main limitation to relativistic spectroscopy in this moment is due to the uncertainties in the physics, not to the signal-to-noise of the data.

The best-fit nuclear column density varies between 2.8 and $3.4 \times 10^{22} \mathrm{~cm}^{-2}$, i.e. always in the Compton-thin regime. The obscuring matter covering the nucleus, however, has an averaged integrated column density about two orders of magnitude larger. Given the moderate inclination of the system, this suggests that we are looking at the system through a thin atmosphere grazing the surface of the torus.

Our best-fit inclination angles of AD or torus for NLSy1 NGC 5506 are $40^{\circ}<\theta<50^{\circ}$. However, Decarli et al. (2008) assumed low inclination angles $\left(\lesssim 15^{\circ}\right)$ and flat geometries of broadline regions to explain the smaller FWHMs of broad lines in NLSy1. This assumption is different from our fit results. In this case, the 'narrow' broad emission lines of NGC 5506 can only be attributed to its smaller SMBH mass, which agrees with the study of Nikołajuk et al. (2009) supporting smaller $M_{\mathrm{BH}}$ of NGC 5506 .

\section{ACKNOWLEDGMENTS}

This work was supported by the NSFC (Grant No. U1531117 and 11305038), Fudan University (Grant No. IDH1512060), and the Thousand Young Talents Program. S.S. also acknowledges support from the AHEAD program for his visit to INAF-Bologna in June 2016. C.B. also acknowledges support from the Alexander von Humboldt Foundation. In this work, the authors used the data supplied by NASA's High Energy Astrophysics Science Archive Research Center in the US. The authors also used the data products from the XMM-Newton, Suzaku, NuSTAR, and Chandra, funded by the ESA, JAXA, NASA.

\section{REFERENCES}

Arnaud K. A., 1996, in Jacoby G. H., Barnes J., eds, Astronomical Society of the Pacific Conference Series Vol. 101, Astronomical Data Analysis Software and Systems V. p. 17

Ballantyne D. R., Vaughan S., Fabian A. C., 2003, MNRAS, 342, 239

Bambi C., 2015, preprint, (arXiv:1509.03884)

Bardeen J. M., Press W. H., Teukolsky S. A., 1972, ApJ, 178, 347

Barr P., White N. E., Page C. G., 1985, MNRAS, 216, 65P

Bianchi S., Balestra I., Matt G., Guainazzi M., Perola G. C., 2003, A\&A, 402, 141

Bianchi S., Miniutti G., Fabian A. C., Iwasawa K., 2005, MNRAS, 360, 380

Bond I., Matsuoka M., Yamauchi M., 1993, The Astrophysical Journal, 405, 179

Brenneman L. W., Reynolds C. S., 2006, ApJ, 652, 1028

Cash W., 1979, ApJ, 228, 939

Dauser T., García J., Parker M. L., Fabian A. C., Wilms J., 2014, MNRAS, 444, L100

Dauser T., García J., Walton D. J., Eikmann W., Kallman T., McClintock J., Wilms J., 2016, A\&A, 590, A76

Decarli R., Dotti M., Fontana M., Haardt F., 2008, MNRAS, 386, L15

Di Matteo T., 1998, MNRAS, 299, L15

Fabian A. C., Rees M. J., Stella L., White N. E., 1989, MNRAS, 238, 729

Fabian A. C., et al., 2009, Nature, 459, 540

Gabriel C., et al., 2004, in Ochsenbein F., Allen M. G., Egret D., eds, Astronomical Society of the Pacific Conference Series Vol. 314, Astronomical Data Analysis Software and Systems (ADASS) XIII. p. 759

Galeev A. A., Rosner R., Vaiana G. S., 1979, ApJ, 229, 318

García J., et al., 2014, ApJ, 782, 76

George I. M., Fabian A. C., 1991, MNRAS, 249, 352

González Delgado R. M., Heckman T., Leitherer C., 2001, ApJ, 546, 845

Grandi P., Tagliaferri G., Giommi P., Barr P., Palumbo G. G. C., 1992, ApJS, 82,93

Guainazzi M., Bianchi S., 2007, MNRAS, 374, 1290

Guainazzi M., Risaliti G., Nucita A., Wang J., Bianchi S., Soria R., Zezas A., 2009, A\&A, 505, 589

Guainazzi M., Bianchi S., Matt G., Dadina M., Kaastra J., Malzac J., Risaliti G., 2010a, MNRAS, 406, 2013

Guainazzi M., Bianchi S., Matt G., Dadina M., Kaastra J., Malzac J., Risaliti G., 2010b, Monthly Notices of the Royal Astronomical Society, 406, 2013

Haardt F., Maraschi L., 1991, ApJ, 380, L51

Haardt F., Maraschi L., Ghisellini G., 1994, ApJ, 432, L95

Huenemoerder D. P., et al., 2011, AJ, 141, 129

Jiang J., Bambi C., Steiner J. F., 2015, ApJ, 811, 130

Kaastra J. S., Bleeker J. A. M., 2016, A\&A, 587, A151

Kallman T., Bautista M., 2001, ApJS, 133, 221

King A., 2005, ApJ, 635, L121

Kinkhabwala A., et al., 2002, ApJ, 575, 732

Komossa S., Xu D., 2007, ApJ, 667, L33

Laor A., 1991, ApJ, 376, 90

Lohfink A. M., Reynolds C. S., Miller J. M., Brenneman L. W., Mushotzky R. F., Nowak M. A., Fabian A. C., 2012, ApJ, 758, 67

Madsen K. K., et al., 2015, ApJS, 220, 8

Marinucci A., Bianchi S., Nicastro F., Matt G., Goulding A. D., 2012, ApJ, 748,130

Matt G., et al., 2015, MNRAS, 447, 3029

McHardy I. M., Koerding E., Knigge C., Uttley P., Fender R. P., 2006, Nature, 444, 730

Merloni A., Fabian A. C., 2001, MNRAS, 328, 958

Miller J. M., 2007, ARA\&A, 45, 441 
Murphy K. D., Yaqoob T., 2009, MNRAS, 397, 1549

Nagar N. M., Oliva E., Marconi A., Maiolino R., 2002, A\&A, 391, L21

Nandra K., Pounds K. A., 1994, MNRAS, 268, 405

Nandra K., Pounds K. A., Stewart G. C., Fabian A. C., Rees M. J., 1989, MNRAS, 236, 39P

Nandra K., O’Neill P. M., George I. M., Reeves J. N., 2007, MNRAS, 382, 194

Nardini E., Fabian A. C., Walton D. J., 2012, MNRAS, 423, 3299

Netzer H., 2013, The Physics and Evolution of Active Galactic Nuclei

Nikołajuk M., Czerny B., Gurynowicz P., 2009, MNRAS, 394, 2141

Parker M. L., et al., 2014, MNRAS, 443, 1723

Pounds K. A., Page K. L., 2005, MNRAS, 360, 1123

Pounds K. A., Nandra K., Stewart G. C., George I. M., Fabian A. C., 1990, Nature, 344, 132

Reynolds C. S., 2014, Space Sci. Rev., 183, 277

Reynolds C. S., Fabian A. C., 2008, ApJ, 675, 1048

Reynolds C. S., Nowak M. A., 2003, Phys. Rep., 377, 389

Risaliti G., et al., 2013, Nature, 494, 449

Sako M., Kahn S. M., Paerels F., Liedahl D. A., 2000, ApJ, 543, L115

Sambruna R. M., Netzer H., Kaspi S., Brandt W. N., Chartas G., Garmire G. P., Nousek J. A., Weaver K. A., 2001, ApJ, 546, L13

Schurch N. J., Warwick R. S., Griffiths R. E., Kahn S. M., 2004, MNRAS, 350,1

Smith R. K., Brickhouse N. S., Liedahl D. A., Raymond J. C., 2001, ApJ, 556, L91

Storchi-Bergmann T., Fernandes R. C., Schmitt H. R., 1998, ApJ, 501, 94

Strüder L., et al., 2001, A\&A, 365, L18

Tan Y., Wang J. X., Shu X. W., Zhou Y., 2012, ApJ, 747, L11

Turner M. J. L., et al., 2001, A\&A, 365, L27

Uttley P., 2007, in Ho L. C., Wang J.-W., eds, Astronomical Society of the Pacific Conference Series Vol. 373, The Central Engine of Active Galactic Nuclei. p. 149

Viegas-Aldrovandi S. M., Contini M., 1989, ApJ, 339, 689

Walton D. J., Reis R. C., Cackett E. M., Fabian A. C., Miller J. M., 2012, MNRAS, 422, 2510

Walton D. J., Nardini E., Fabian A. C., Gallo L. C., Reis R. C., 2013, MNRAS, 428, 2901

Wang T., Mihara T., Otani C., Matsuoka M., Awaki H., 1999, ApJ, 515, 567

Young A. J., Wilson A. S., Shopbell P. L., 2001, ApJ, 556, 6

Zoghbi A., Fabian A. C., Uttley P., Miniutti G., Gallo L. C., Reynolds C. S., Miller J. M., Ponti G., 2010, MNRAS, 401, 2419

Zoghbi A., Reynolds C., Cackett E. M., Miniutti G., Kara E., Fabian A. C., 2013, ApJ, 767, 121 